\title{
CONCEPTUAL DESIGN OF THE PEFP RAPID CYCLING SYNCHROTRON*
}

\author{
B. Chung", Y. S. Cho, PEFP, KAERI, Korea \\ Y. Y. Lee, BNL, Upton, New York, 11973, U.S.A.
}

\section{Abstract}

The Proton Engineering Frontier Project (PEFP) proposes the $1-2 \mathrm{GeV}$ rapid cycling synchrotron accelerator as an extension of the PEFP linac, which initially is a $15 \mathrm{~Hz}$ rapid-cycling synchrotron (RCS) with the injection energy of $100 \mathrm{MeV}$. The target beam power is $58 \mathrm{~kW}$ at $1.0 \mathrm{GeV}$ beam energy in the first stage, through 5 stages of improvement it could be reach to $900 \mathrm{~kW}$. The PEFP RCS is basically composed of magnet lattice of main ring, injection system and extraction system. The conceptual lattice design of the RCS as well as the simulations of an injection system is described in this paper.

\section{INTRODUCTION}

The Proton Engineering Frontier Project (PEFP) is a research project to develop a $100 \mathrm{MeV}, 20 \mathrm{~mA}$ pulsed proton linear accelerator to be used in basic/applied scientific R\&D programs and industrial applications [1]. The PEFP proposes the $1-2 \mathrm{GeV}$ Rapid Cycling Synchrotron (RCS) accelerator as an extension of the PEFP linac, which can be used for nuclear and highenergy physics experiment, spallation neutron source, radioisotope, medical research, etc. The spallation neutron source require high power and high current proton beam, but the radioisotope production and medical research facilities need relatively low energy and low current proton beam. The PEFP RCS with the extraction energy $1-2 \mathrm{GeV}$ and injection energy 0.1 $0.2 \mathrm{GeV}$ is able to optimally support both the spallation neutron source and radioisotope production and medical research facility. The fast extraction system of PEPF RCS machine can be a spallation neutron source facility and the slow extraction system can be a radioisotope production and medical research facility simultaneously. The machine capability could be improved through an improvement project later. The current power upgrade plan of the PEFP RCS ring is presented in Table 1.

Table 1: Power upgrade plan

\begin{tabular}{|c|c|c|c|c|c|}
\hline Stage & $\begin{array}{c}\text { Injectio } \\
\mathrm{n} \\
\text { energy } \\
{[\mathrm{MeV}]}\end{array}$ & $\begin{array}{c}\text { Ejection } \\
\text { energy } \\
{[\mathrm{GeV}]}\end{array}$ & $\begin{array}{c}\text { Repetit } \\
\text { ion rate } \\
{[\mathrm{Hz}]}\end{array}$ & $\begin{array}{c}\mathrm{RF} \\
\text { voltage } \\
{[\mathrm{kV}]}\end{array}$ & $\begin{array}{c}\text { Beam } \\
\text { power } \\
{[\mathrm{kW}]}\end{array}$ \\
\hline Step 1 & 100 & 1 & 15 & 45 & 58 \\
\hline Step 2 & 100 & 1 & 30 & 90 & 116 \\
\hline Step 3 & 100 & 2 & 30 & 130 & 232 \\
\hline Step 4 & 100 & 2 & 60 & 260 & 466 \\
\hline Step 5 & 200 & 2 & 60 & 260 & 900 \\
\hline
\end{tabular}

*This work was supported by the 21C Frontier R\&D program sponsored by Ministry of Science and Technology, Korean Government.

\#cbc0726@kaeri.re.kr
In this paper, the conceptual design of the PEFP RCS for a pulsed proton beam is proposed. A basic lattice design and a conceptual design for the injection system as well as an extraction system are described.

\section{LATTICE AND LAYOUT}

A basic lattice structure is determined by the disposal of magnets such as dipole, quadrupole, sextupole, etc. And the lattice magnet specifications generally depend on the extraction beam energy. The PEFP RCS lattice has a four-fold symmetry. Although the low-order machine symmetry can give a dangerous low-order structure resonance, two or four symmetric lattice structure is usually accepted to ensure the space for other essential facilities [2]. The PEFP RCS has a four superperiodicity and a 20 pseudo-periodicity. The lattice of the straight section as well as the arc section is constituted of simple FODO cells.

Achromatic structure is used at the arc section to obtain a dispersion-free long straight section for establishing the injection and extraction system. Machine tune is 4.39 for $\mathrm{x}$-direction and 4.29 for $\mathrm{y}$-direction, which can avoid the crucial structure resonance. The schematic layout of the PEFP RCS machine is shown in Fig. 1.

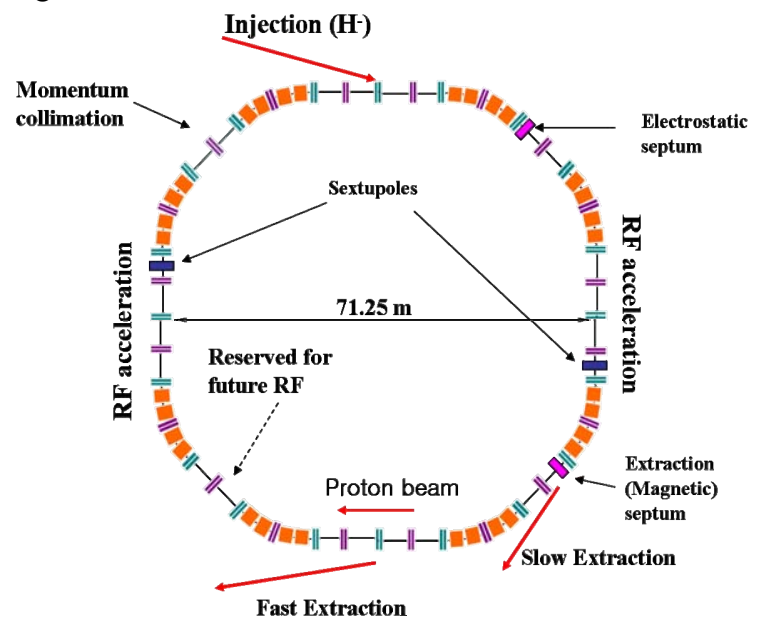

Figure 1: Lattice Layout for the PEFP RCS.

Figure 2 shows the beta functions and dispersion function for a super-period including the arc section of the PEFP RCS. Usually the momentum collimation is accomplished at the center of the arc section because of large momentum dispersions in this area. Crucial parameters regarding the PEFP RCS design are represented in Table 2. 


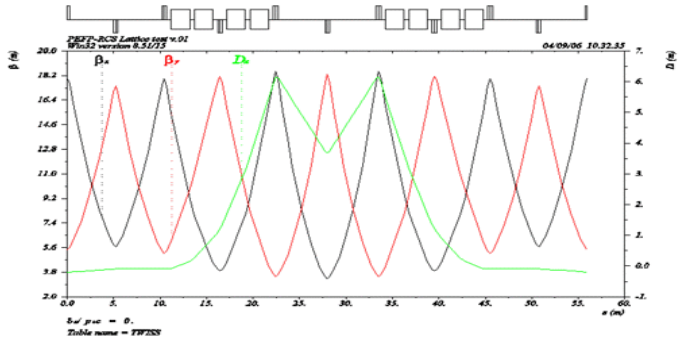

Figure 2: Beta function and dispersion function for a super-period of the PEFP RCS.

\section{INJECTION SYSTEM}

The injection system from the linac to main ring is very important part in the entire accelerator system because most of the beam losses occur in this region. The PEFP injection system use the way of a charge exchange multi-turn injection, locate in the dispersion free long straight section. Figure 3 shows the schematic layout of the PEFP injection system and four kicker magnets for the horizontal $(\mathrm{H})$ orbit bump and other four kicker magnets for vertical (V) orbit bump are used. The height of the injection chicane is about $6 \mathrm{~cm}$ in the transverse plane. Transverse painting controls the uniformity of the phase space distribution and the shape of the beam profile and facilitates the fundamental space charge limit. In the transverse plane, either correlated or anticorrelated relation of the horizontal and vertical bumps produce the different beam profiles, which are correlated painting and anti-correlated painting respectively [3]. The PEFP painting scheme is a correlated painting and the injection protons are about $2.42 * 10^{13}$ for the space charge tune shift -0.2 .

Table 2: Basic Parameters of the PEFP RCS

\begin{tabular}{|l|l|}
\hline Injected particle & H- \\
\hline Injection energy $(\mathrm{GeV})$ & $0.1 \sim 0.2$ \\
\hline Extraction energy $(\mathrm{GeV})$ & $1 \sim 2$ \\
\hline Repetition rate Fast/Slow $(\mathrm{Hz})$ & $15 \sim 30 \sim 60 / 1$ \\
\hline Beam power $(\mathrm{kW})$ & $58 \sim 900$ \\
\hline Admittance $(\mathrm{mm} . \mathrm{mrad})$ & 560 \\
\hline Beam emittance $(\mathrm{mm} . \mathrm{mrad})$ & 280 \\
\hline Laslett tune shift & -0.2 \\
\hline Circumference $(\mathrm{m})$ & 223.824 \\
\hline Lattice structure & FODO \\
\hline Super-period & 4 \\
\hline Number of cell & 20 \\
\hline Machine tune[Qx, Qy] & $4.39 / 4.29$ \\
\hline Transition & 4.4 \\
\hline Number of dipoles & 32 \\
\hline RF harmonic & 2 \\
\hline
\end{tabular}

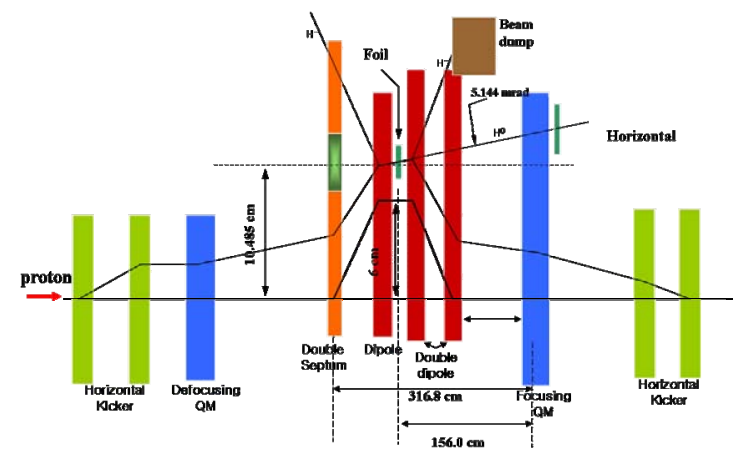

Figure 3: PEFP Injection Layout.

\section{EXTRACTION SYSTEM}

The PEFP extraction system consists of two different facilities, one is a fast extraction system for the spallation neutron source or other high power experiments and the other one is a slow extraction system for the radioisotope production or the medical application and so on. The magnet cycle distribution can determine the basic difference between the fast extraction and slow extraction. In the case magnetic cycle includes a long flat top at the top of the cycle where the slow extraction occurs. For example, figure 4 presents a repetition rate of a slow extraction, which is a magnetic cycle with a long flat top at the desired energy and with a short acceleration and deceleration time.

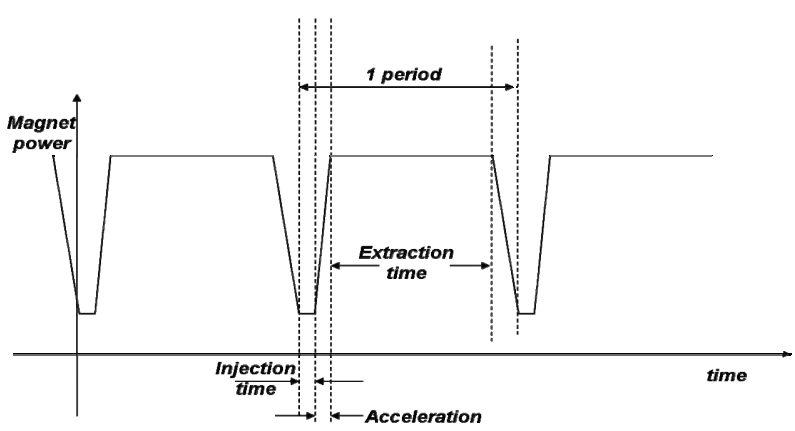

Figure 4: Illustration of a slow extraction cycle.

Figure 5 shows the PEFP fast extraction system which consists of 5-6 kicker magnets and one septum magnet. The required voltage at $2 \mathrm{GeV}$ extraction is $37 \mathrm{kV}$ for each kicker magnet when the extraction displacement is $82.6 \mathrm{~mm}$ at the septum magnet. A small programmable bump is used to alleviate beam extraction.

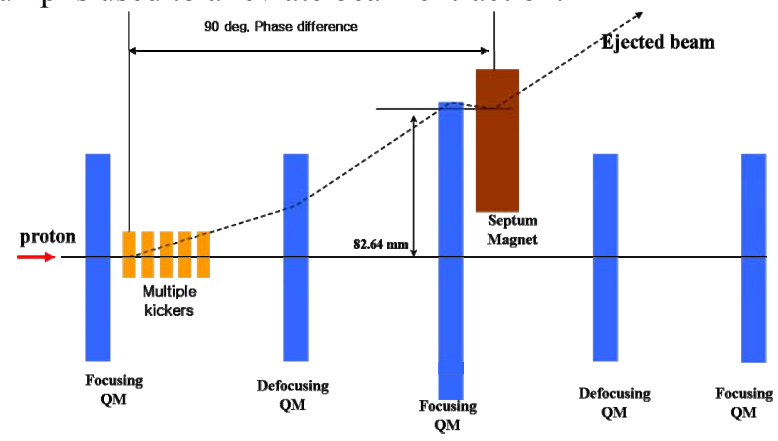

Figure 5: PEFP Fast Extraction System. 
A slow extraction can be achieved by the resonance which is caused by the closed orbit distortion via quadruole or sextupole errors [4]. The PEFP slow extraction is accomplished by the third order resonances of the sextupole magnets. The conceptual layout is given in Fig. 6. Both the electrostatic septum and the magnetic septum are used to reduce the beam loss at the extraction point. Two sextupole magnets are symmetrically located among the ordinary lattice magnets to control the third order resonance. The extracting beam current can be controlled by tuning the stop band width of the sextupole or by adjusting the tune of the machine.

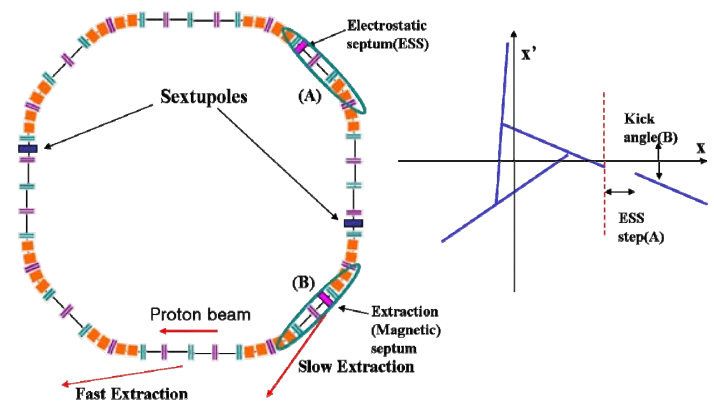

Figure 6: PEFP Slow Extraction, (A) Electrostatic Septum and (B) Magnetic Septum.

\section{CONCLUSION}

The conceptual design of the Rapid Cycling Synchrotron as an extension of the PEFP linac has been accomplished. The proton accelerator from $0.1-0.2 \mathrm{GeV}$ to $1-2 \mathrm{GeV}$ has been considered as well as the injection and extraction system. In the initial stage, the target beam power with $15 \mathrm{~Hz}$ repetition rate is about $58 \mathrm{~kW}$ at $1 \mathrm{GeV}$ extraction. The $\mathrm{H}^{-}$charge exchange injection is chosen in the dispersion free straight section as an injection method of PEFP RCS. The characteristic feature of the PEFP RCS is that it is applicable to the spallation neutron source facility via fast extraction and the medical research as well as the radioisotope production facility via slow extraction.

\section{REFERENCES}

[1] B. H. Choi, Status of the PEFP, Proceedings of PAC05 (Knoxville, TN, 2005), p. 576.

[2] J. Wei, Synchrotron and accumulators for highintensity proton beams, Rev. Mod. Phys, Vol. 75, 2003, p. 1383.

[3] Y. Suzuki, I. Yamane, Workshop on Injection and Extraction, Abingdon (1999).

[4] E. J. N. Wilson, Proton Synchrotron Accelerator Theory, CERN 77-07, 1977. 